\title{
Probing TeV scale physics via ultra cold neutron decays and calculating non-standard baryon matrix elements
}

\author{
Rajan Gupta* ${ }^{\dagger}$ Tanmoy Bhattacharya and Anosh Joseph \\ Theoretical Division, Los Alamos National Laboratory, Los Alamos, NM 87545, USA
}

Huey-Wen Lin and Saul D. Cohen

Department of Physics, University of Washington, Seattle, WA 98195

\begin{abstract}
We motivate undertaking precision analyses of neutron decays to look for signatures of new scalar and tensor interactions that can arise in extensions of the Standard Model at the TeV scale. The key ingrediant needed to connect experimental data with theoretical analysis are high-precision calculations of matrix elements of isovector bilinear operators between the decaying neutron and final state proton. We describe the status of our Lattice QCD program of using valence clover fermions on dynamical $N_{f}=2+1+1$ HISQ configurations generated by the MILC Collaboration. On the theoretical side we use the effective field theory method and provide both model independent and dependent analyses to obtain bounds on possible scalar and tensor interactions, both from low energy experiments and LHC data.
\end{abstract}

The XXIX International Symposium on Lattice Field Theory

July 10-16, 2011

Lake Tahoe, California

*Speaker.

†A-UR-12-10238 


\section{Introduction}

New physics at a given high energy scale, such as that generated by the exchange of any heavy boson, gives rise to measurable effects at the hadronic scale. The most well known example is weak interactions (WI) mediated by the exchange of $W$ and $Z$ vector bosons that are responsible, for example, for neutron $\beta$-decay. In the 1930s, Fermi parameterized the charged current decay in terms of an effective four-fermion interaction. This effective theory was also able to explain muon decay in terms of a single coupling constant $G_{F}$. Elucidation of the Lorentz structure of the interaction, $V-A$, came in 1957 with the work of Marshak and Sudershan, and Feynman and Gell-Mann. Until then, physicists had considered scalar and tensor interactions as viable candidates. Analysis of low energy decays revealed a number of properties of WI, such as quark-lepton universality, and the weak scale, $\sim 100 \mathrm{GeV}$, related to the mass of the $W$ and $Z$ vector bosons that are now part of the electroweak theory. In formulating a consistent electroweak theory, it was also clear that associated with the weak scale there has to be a second scale, $O(\mathrm{TeV})$, characterizing the breaking of electro-weak symmetery since at the hadronic scale WI are $10^{-5}$ times smaller than the electric force.

To break the electro-weak symmetery, explain the origin of masses of quarks, leptons and weak bosons (Higgs phenomena) and to stabilize the Standard Model against radiative corrections motivates the need for new physics at the TeV scale. The LHC has, in fact, been designed to probe new physics at this scale. To explore the $1-10 \mathrm{TeV}$ range, and re-examine the possibility of scalar and tensor interactions, we follow the complementary approach to direct detection at the LHC by looking for deviations from the SM in precision experiments at the hadronic scale. This talk gives an overview of the combined experimental and theoretical precision analyses of ultracold neutron (UCN) decays required to probe novel interactions at the TeV Scale. In particular, we would like to address the following questions:

- Are there novel scalar or tensor interactions at the TeV scale?

- What are the respective coupling constants and what bounds can be put on them?

- What are their manifestations at the hadronic scale and what decays/processes are the best candidates for investigating them or for setting upper bounds on their strengths?

- What beyond the SM candidate theories can accomodate these new interactions and satisfy all other known constraints?

Decays of UCN are interesting for probing new physics because there are terms in the neutron decay distribution that are particularly sensitive to scalar and tensor interactions at the $10^{-3}$ level. In these terms in the decay distribution, the SM contributions are suppressed due to the helicity flip factor $m_{e} / E_{e}$, so they are of the same order as the signal. The details of the theoretical framework, analysis and preliminary results are given in Ref. [1], and we will follow the notation established there. In this talk we summarize the key points of the formalism and give some preliminary results. A discussion of the Lattice QCD part of the project will be presented by Lin [2] and of the exisiting bounds from other low energy experiments and from the LHC by Bhattacharya [3]. 


\section{Neutron decay}

The differential neutron decay distribution function $D\left(E_{e}, \mathbf{p}_{e}, \mathbf{p}_{v}, \sigma_{n}\right)$, keeping only terms relevant to the discussion here, can be written as $[4,1]$

$$
D\left(E_{e}, \mathbf{p}_{e}, \mathbf{p}_{v}, \sigma_{n}\right)=1+c_{0}+c_{1} \frac{E_{e}}{M_{N}}+\frac{m_{e}}{E_{e}} \bar{b}+\bar{B}\left(E_{e}\right) \frac{\sigma_{n} \cdot \mathbf{p}_{v}}{E_{v}}+\cdots,
$$

where $\mathbf{p}_{e}$ and $\mathbf{p}_{v}$ denote the electron and neutrino three-momenta, and $\sigma_{n}$ denotes the neutron polarization. Here $\bar{b} \equiv b^{S M}+B^{B S M}$ is an effective Fierz interference term and $\bar{B}\left(E_{e}\right)$ is an effective energy-dependent correlation coefficient given by

$$
\bar{B}\left(E_{e}\right)=B_{\mathrm{LO}}(\tilde{\lambda})+c_{0}^{(B)}+c_{1}^{(B)} \frac{E_{e}}{M_{N}}+\frac{m_{e}}{E_{e}}\left(b_{v}^{\mathrm{SM}}+b_{v}^{\mathrm{BSM}}\right)
$$

The terms most sensitive to novel scalar and tensor interactions are proportional to the helicityflip factor $\frac{m_{e}}{E_{e}}$, and characterized by the parameters $\bar{b}$ and $b_{v}^{\mathrm{SM}}+b_{v}^{\mathrm{BSM}}$. The beyond the Standard Model (BSM) contributions to these parameters arise from novel scalar and tensor interactions (with coupling constants $\varepsilon_{S}$ and $\varepsilon_{T}$ ) generated at the TeV scale. To extract $\bar{b}$ requires precison measurements of the electron spectrum, while $\left(b_{v}^{\mathrm{SM}}+b_{v}^{\mathrm{BSM}}\right)$ requires measuring the asymmetry between the neutron polarization and the anti-neutrino momentum.

Current bounds on $b^{B S M}$ and $b_{v}^{\mathrm{BSM}}$ leave open the possibility that these could be as large as $10^{-3}$, which implies that the scale for these novel interactions is TeV. Standard Model contributions, $b^{\mathrm{SM}}$ and $b_{v}^{\mathrm{SM}}$, are $O\left(10^{-3}\right)$ and known to $O\left(10^{-5}\right)$. So the sought after signal is the same size as the well-understood SM background. Two experiments at the Los Alamos ultracold neutron source, called UCNb [5] and UCNB [6], are being carried out by our experimental colleagues to measure these parameters at the $10^{-3}$ level. In a UCN decay the recoil effects, $\left(p_{n}-p_{p}\right) / M_{N} \sim 10^{-3}$, are small and of the same size as the signal while there are no nuclear effects. Even though the signal in UCN decays is not swamped by the background, nevertheless, the experiments are very challenging.

\section{UCNb and UNCB experiments}

The two separate experiments, UCNb and UNCB, will use the same UCN source. The setup is shown in Fig. 1. The proposed plan is to make $10^{-3}$ measurements of $\bar{b}$ and $\bar{b}-\left(b_{v}^{\mathrm{SM}}+b_{v}^{\mathrm{BSM}}\right)$ by the end of 2013 and based on the results and detector performance and sensitivity achieved propose a $10^{-4}$ level experiment to DOE.

The goal of the UCNb experiment is a high precision measurement of the electron decay spectrum. In Fig. 2 we show the full SM expected spectrum $F_{0}\left(E_{e}\right)$ of the electron in the limit of zero nuclear recoil (left panel) and the deviation from it assuming $b^{B S M}=10^{-3}$ (right panel). To achieve the design sensitivity requires building a very sensitive and high resolution calorimeter. The UCNB experiment measures the asymmetry between the momentum direction of the neutrino and the spin of the decaying neutron and yields $\bar{b}-\left(b_{v}^{\mathrm{SM}}+b_{v}^{\mathrm{BSM}}\right)$. The neutrino momentum is determined indirectly by measuring the electron and proton momenta in coincidence. (In practice, the precision possible on these momenta is not sufficient to fully reconstruct the neutrino momentum so a reduced-detail observable is used from which $\bar{b}-\left(b_{v}^{\mathrm{SM}}+b_{v}^{\mathrm{BSM}}\right)$ will be extracted [1].) 
$40 \mathrm{ucn} / \mathrm{cc}$

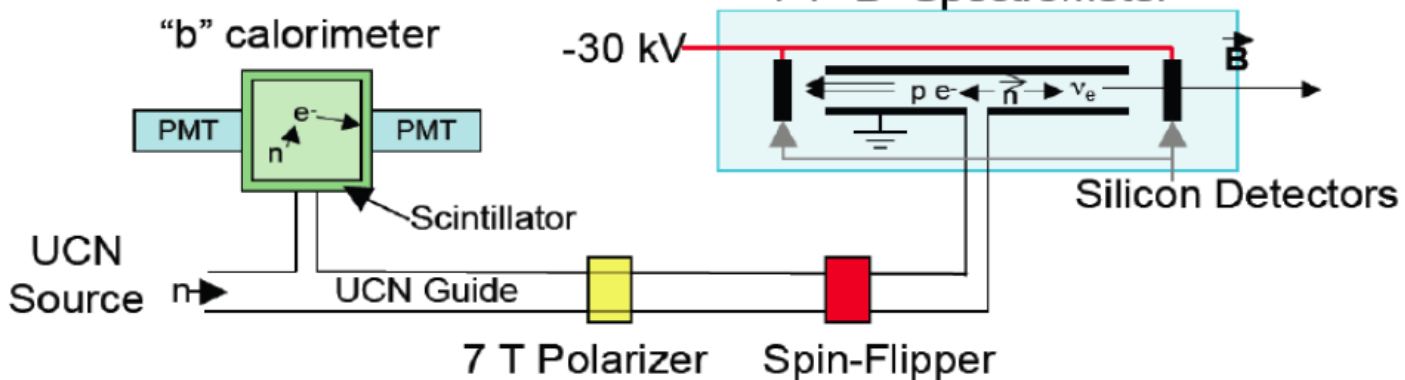

Figure 1: The experimental set up for UCNb (left panel) and UCNB (right panel). The UCNb experiment is a calorimeter that measures the spectrum of the electron. In the UCNB experiment the UCN enter the spectrometer after being polarized to over $99.5 \%$. The asymmetry between the momentum direction of the neutrino and the spin of the decaying neutron is measured indirectly by detecting coincidences between the proton and the electron. The analysis is more complex and what one extracts in the end is $\bar{b}-\left(b_{v}^{\mathrm{SM}}+b_{v}^{\mathrm{BSM}}\right)$.
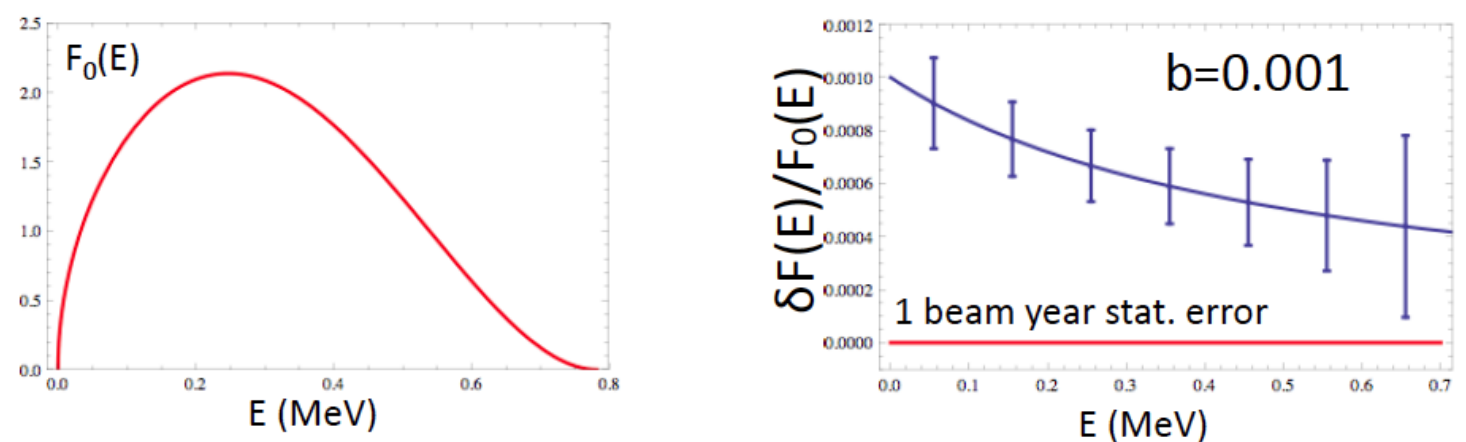

Figure 2: The left panel shows a plot of the electrum spectrum $F_{0}(E)$. The right panel shows the expected deviations assuming $\bar{b}=0.001$, and the statistical errors with one year of running.

\section{Existing constraints}

In Fig. 3, we illustrate how precision measurements of $b$ and $b_{v}$ at the $10^{-3}$ level can reveal the existence of new physics with mass scale $\Lambda_{i}$ in the multi-TeV range, which will be explored directly at the LHC. Furthermore, the two panels highlight the difference in bounds between using previous phenomenological estimates for $g_{S}$ and $g_{T}$ [7] and current LQCD estimates [1,2]. In these figures, $b_{0^{+}}$represents the existing constraint from $0^{+} \rightarrow 0^{+}$nuclear beta decays. While the LHC will be able to produce $W^{\mathrm{BSM}}$ with masses in the $0.2-3 \mathrm{TeV}$ range and may provide precise measurements of their masses, the proposed neutron decay experiments will provide the most precise measurement of their spin and couplings, needed to reconstruct the $\mathrm{TeV}$-scale theory that will replace the SM. Moreover, even if $W^{\mathrm{BSM}}$ is out of the kinematic reach of the LHC (couplings are $O\left(10^{-4}\right)$ or smaller) or swamped by background, the interactions that it generates may lead to observable effects in the proposed neutron-decay experiments.

To connect the new theory (the theory replacing the SM at the TeV scale) to low-energy experiments requires the calculation of the matrix elements of the new interactions between the decaying 
neutron and the final state proton. Even through the new theory is unknown, its consequences at the hadronic scale can be evaluated in terms of an effective theory. In this model-independent effective theory, the leading operators encapsulating novel scalar and tensor interactions are iso-vector quark bilinear operators $\bar{u} d$ and $\bar{u} \sigma^{\mu v} d$. Knowing their matrix elements at $q \equiv p_{n}-p_{p}=0$, i.e. the scalar and tensor charges $g_{S}$ and $g_{T}$, will allow us to put bounds on the couplings $\varepsilon_{S, T}$. The best method for calculating these matrix elements with control over all systematic errors is Lattice QCD. An overview of our ongoing lattice calculations is given next.

\section{Lattice QCD Calculations}

In Ref. [1], we show that to interpret experimental results at the $10^{-3}$ level requires estimating $g_{S}$ and $g_{T}$ with 10-20\% precision. In Fig. 4, taken from Ref. [1] and discussed further in the talk by Bhattacharya [3], we show the allowed region in the $\varepsilon_{S, T}$ plane as a function of the uncertainty in $g_{S}$ and $g_{T}$ assuming an experimental accuracy of $10^{-3}$. Need for higher precision beyond 10$20 \%$ in $g_{S}$ and $g_{T}$ will require higher precision in experiments. Thus, the anticipated experimental precision sets the goal of our LQCD calculations for the 2013-2014 timeframe.

As discussed further in the talk by Lin [2], 10-20\% precision in $g_{S}$ and $g_{T}$ requires control over the extrapolations in quark mass to the physical pion mass and in lattice spacing to the continuum limit, and non-perturbative determination of the renormalization constants of the operators. To control these extrapolations requires high statistics data at three or more values of the lattice spacing and light quark masses. To satisfy these requirements within the time frame of the experiments, three years, motivated us to use the $2+1+1$ flavor dynamical gauge configurations generated by
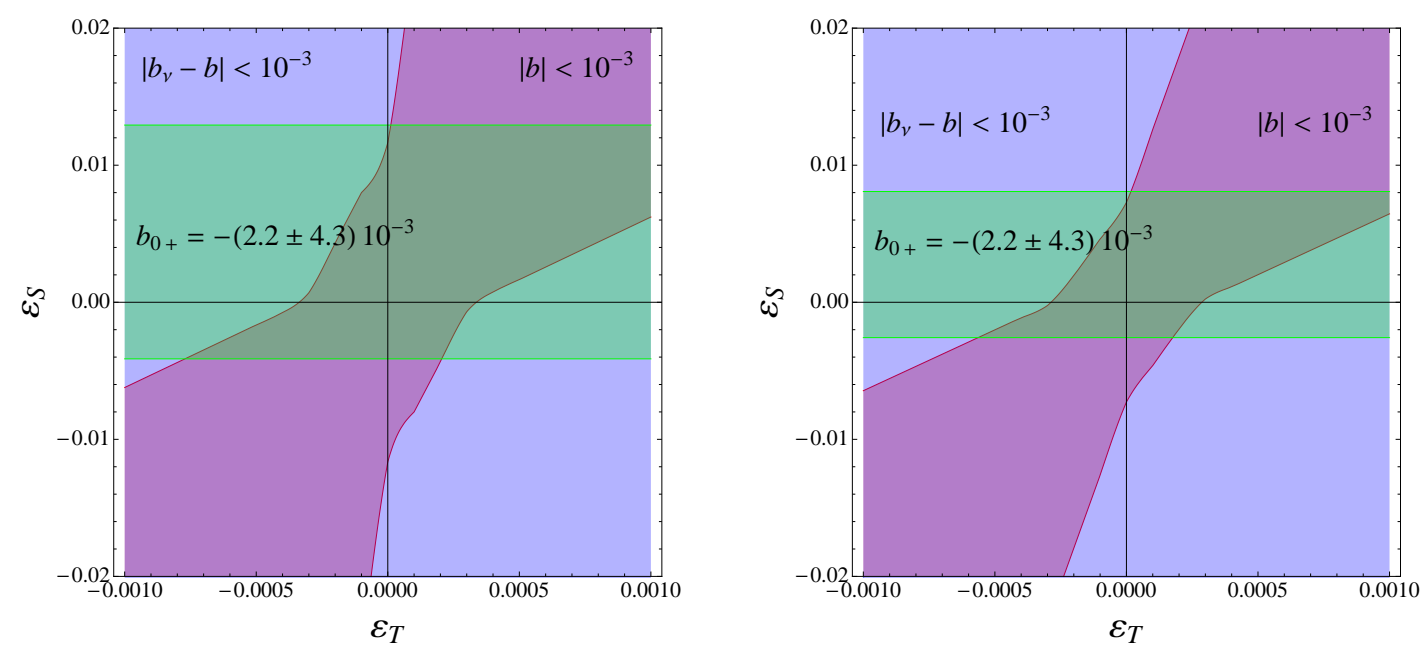

Figure 3: Left panel: $90 \%$ Confidence Level (C.L.) allowed regions in the $\varepsilon_{S^{-}} \varepsilon_{T}$ plane implied by (i) the existing bound on $b_{0^{+}}$characterizing the $0^{+} \rightarrow 0^{+}$nuclear beta decays (green horizontal band); (ii) projected measurements of $\bar{b}$ and $\left(b_{v}^{\mathrm{SM}}+b_{v}^{\mathrm{BSM}}\right)-\bar{b}$ in neutron decay (red bow-tie shapes and blue regions) at the $10^{-3}$ level; (iii) hadronic matrix elements taken in the ranges $0.25<g_{S}<1.0,0.6<g_{T}<2.3$ [7]. Right panel: same as left panel but with scalar and tensor charges taken from Lattice QCD: $g_{S}=0.8(4)$ and $g_{T}=1.05(35)[1]$. Note that the constraint from $b_{0^{+}}$also improves with higher precision in $g_{S}$. The effective couplings $\varepsilon_{S, T}$ are defined in the $\overline{\mathrm{MS}}$ scheme at $2 \mathrm{GeV}$. 


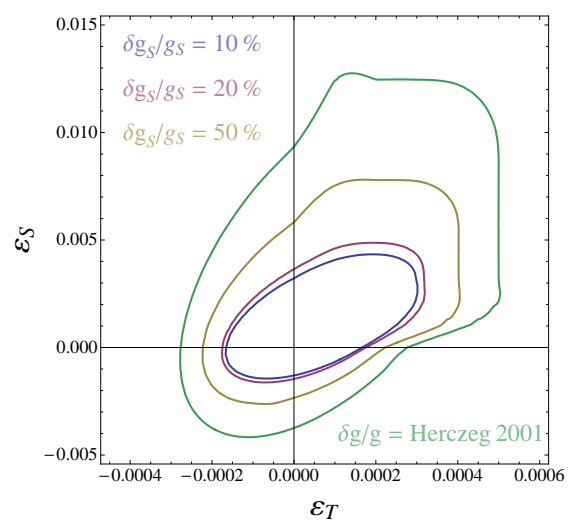

Figure 4: Constraints over the allowed regions in the $\varepsilon_{S}, \varepsilon_{T}$ plane as a function of the uncertainty in $g_{S}$ and $g_{T}$ for different values of $\delta g_{S} / g_{S}$ assuming $\delta g_{T}=2 / 3 \delta g_{S}$. The outer contour is based on the uncertainties in $g_{S}$ and $g_{T}$ used in the phenomenological analysis by Herczeg [7]. The convergence of the contours show that for an experimental uncertainty of $10^{-3}$, the goal of LQCD calculations should be to calculate $g_{S}$ and $g_{T}$ to $10-20 \%$ accuracy.

the MILC Collaboration using the HISQ action. At the end of 2011, nine ensembles, each of about 1000 lattices, at the three lattice spacings $0.12,0.09$ and $0.06 \mathrm{fm}$ and each with the light quark mass corresponding to a pion mass of 310, 220 and $140 \mathrm{MeV}$ had already been generated. Furthermore, the generation of these lattices is continuing and supported by the USQCD national program.

For valence quarks we chose to use the improved clover fermion action since the signal in baryon correlators with staggered fermions is poor. Using clover valence quarks on HISQ lattices, however, introduces the well-known problem of exceptional configurations. These are configurations in which the clover Dirac operator has near zero modes. When the valence and sea quark actions are the same, such configurations are suppressed by a concommitant vanishing of their weight in the path integral. Unchecked, exceptional configurations would make anomalously large contributions and degrade the reliability of the ensemble average. In our calculations with cloveron-HISQ, such a cancellation is not guaranteed and checks are required for each ensemble. Our tests, to date, on ensembles with pion masses of 310 and $220 \mathrm{MeV}$, do not show evidence of exceptional configurations. Preliminary simulations at $140 \mathrm{MeV}$ suggest that this fortutious circumstance will most likely not hold at the physical pion mass, so we have restricted our calculations to quark masses with 310 and $220 \mathrm{MeV}$ pions. The analysis of the data presented by Lin [2] shows that data at 310 and $220 \mathrm{MeV}$ already reduces the uncertainty due to the chiral extrapolation very significantly. Nevertheless, in light of the problem with exceptional configurations, our long-term strategy for simulations at the physical quark mass is to perform clover-on-clover calculations.

Another potential source of systematic errors in LQCD calculations is guaranteeing that the matrix elements are calculated between ground states of the proton and the neutron. Mixing with excited states arises because lattice operators automatically couple to the ground state and all its excited states with the same quantum numbers. There are two ways to reduce excited state contamination-design better interpolating operators to enhance overlap with ground state, and increase the separation between the source and sink. We are addressing this problem by using 
smeared sources, doing the calculation with multiple source-sink separations and by explicitly including possible contributions of excited states in our analysis.

Our existing calculations on the 0.12 and 0.09 fm lattices, with pion masses of 310 and 220 $\mathrm{MeV}$ and 400-500 configurations, show that the statistical errors in $g_{S}$ are 5-6 times those in $g_{T}$ and $g_{A}$. The uncertainty in the latter is already at the 5-10\% level. These preliminary studies also show that there is very small dependence of the estimates on the lattice spacing. This gives us confidence that a reliable continuum extrapolation will be possible using data obtained at 0.12 , 0.09 and $0.06 \mathrm{fm}$. We are therefore confident that analyzing the full set of 1000 lattices in each ensemble will yield estimates with 10-20\% accuracy in $g_{S}$ and $g_{T}$. We are also on schedule to reach this milestone over the next two years, in time when the first experimental results are expected.

\section{Acknowledgments}

We thank our collaborators in the theory effort (V. Cirigliano, A. Filipuzzi, M. GonzalezAlonso and M. Graesser) and our experimental colleagues. We thank the MILC Collaboration for sharing the HISQ lattices. These calculations were performed using the Chroma software suite [8]. Numerical simulations were carried out in part on facilities of the USQCD Collaboration, which are funded by the Office of Science of the U.S. Department of Energy, and the Extreme Science and Engineering Discovery Environment (XSEDE), which is supported by National Science Foundation grant number OCI-1053575. The speaker is supported by the DOE grant DE-KA-1401020.

\section{References}

[1] T. Bhattacharya, V. Cirigliano, S. Cohen, A. Filipuzzi, M. Gonzalez-Alonso, M. Graesser, R. Gupta, and H.-W. Lin, Phys. Rev. D (2012), hep-ph/1110.6448.

[2] H.-W. Lin, S. Cohen, , T. Bhattacharya, R. Gupta, and A. Joseph, PoS LATTICE2011, 273 (2011).

[3] T. Bhattacharya, R. Gupta, A. Joseph, S. Cohen, and H.-W. Lin, PoS LATTICE2011, 272 (2011).

[4] H. Abele, Prog.Part.Nucl.Phys. 60, 1 (2008).

[5] K. P. Hickerson, The Fierz Interference Term in Beta-Decay Spectrum of UCN (2009), uCN Workshop, November 6-7 2009, Santa Fe, New Mexico, URL

http://neutron.physics.ncsu.edu/UCN_Workshop_09/Hickerson_SantaFe_2009.pdf.

[6] W. Wilburn et al., Rev. Mex. Fis. Suppl. 55(2), 119 (2009).

[7] P. Herczeg, Prog. Part. Nucl. Phys. 46, 413 (2001).

[8] R. Edwards and B. Joo, Nucl. Phys. Proc. Suppl. 140, 832 (2005), hep-lat/0409003. 